\title{
EFECTO DE LA DISPONIBILIDAD HÍDRICA Y TEMPERATURA SOBRE EL DESPLIEGUE FOLIAR DE GENOTIPOS DE Eucalyptus globulus, Eucalyptus nitens E HÍBRIDOS CON Eucalyptus camaldulensis
}

\author{
Pincheira, Matías ${ }^{12}$ y Rubilar, Rafael ${ }^{13}$
}

\section{RESUMEN}

En especies de rápido crecimiento, el área foliar $(A F)$ permite comparaciones de productividad eficientes entre individuos creciendo bajo condiciones de alta y baja disponibilidad de recursos. Su incremento absoluto se relaciona con factores tales como el agua disponible en el suelo, temperatura, radiación incidente, fotoperiodo y fertilidad del suelo, y se correlaciona directamente con la productividad primaria potencial volumétrica y de biomasa total.

No obstante, a diferencia de lo ocurrido con muchas especies de coníferas, en Eucalyptus el análisis del efecto de la modificación de la disponibilidad de recursos del sitio sobre el despliegue foliar es incipiente, aun cuando el conocer este efecto pudiese minimizar los amplios márgenes de error asociados a los actuales criterios de selección genética, identificando especies, variedades y/o genotipos con mayor flexibilidad y plasticidad ante factores de estrés.

En este contexto, esta investigación analiza el incremento y senescencia foliar de diez genotipos de Eucalyptus, bajo dos condiciones de humedad de suelo, con y sin riego, establecidos en el secano interior de la Región del Bio Bio. El conjunto incluye un E. nitens de origen semilla; seis $E$. globulus, dos de baja, dos de media y dos de alta productividad volumétrica; y tres híbridos (E. nitens $\times$ E. globulus, E. camaldulensis $\times E$. globulus y E. nitens $\times$ E. camaldulensis). Para ello, en una muestra de ramas seleccionadas en la primera temporada de crecimiento en forma no destructiva se cuantificó los incrementos y decrecimientos absolutos y relativos de área foliar, por medio de relaciones alométricas establecidas entre las dimensiones lineales de hoja y rama, y su área foliar individual y total, respectivamente.

El objetivo general del estudio fue establecer la fenología y el efecto de la disponibilidad hídrica y temperatura sobre el despliegue foliar de genotipos de Eucalyptus globulus, E. nitens e híbridos con E. camaldulensis.

Sin riego se identificaron cuatro periodos en el despliegue de superficie fotosintética, incremento inicial después del establecimiento, receso vegetativo, crecimiento de segunda temporada y de senescencia y abscisión foliar. Con riego se observaron los dos primeros periodos, sin ser notoria una reactivación del desarrollo de segunda temporada, y finalmente el cuarto de senescencia. Los individuos del sitio con riego resultaron mucho más sensibles a cambios climáticos, cuya fase de recesión absoluta comenzó 30 días antes. Evaluado el efecto de la temperatura media acumulada $\left({ }^{\circ} \mathrm{C}\right)$ sobre el despliegue foliar de rama $\left(\mathrm{cm}^{2}\right)$, fueron identificados grupos de genotipos con alta y baja sensibilidad a bajas temperaturas.

Palabras clave: Eucalyptus, Rápido crecimiento, Alometría, Estrés hídrico, Sensibilidad al frío.

\footnotetext{
${ }^{12}$ Instituto Forestal, Sede Biobío, Concepción, Chile, mpincheira@infor.cl

${ }^{13}$ Fac. Cs. Forestales, Universidad de Concepción, Concepción, Chile.
} 


\section{SUMMARY}

In fast-growing species, leaf area (AF) enables efficient productivity comparisons between individuals growing under conditions of high and low availability of resources. Its absolute increase is related to factors such as the available water in the soil, temperature, radiation incident, photoperiod, soil fertility, and directly correlates with the potential primary volumetric productivity and total biomass.

However, unlike what occurred with many species of conifers, in Eucalyptus analysis of the effect of changing the availability of site resources on deployment leaf is emerging, although knowing this effect would minimize the wide margins of error current associated with genetic selection criteria, identifying species, varieties and / or genotypes with greater flexibility and plasticity to stress factors.

In this context, this research analyzes the increase and leaf senescence in ten genotypes of Eucalyptus, under two soil moisture conditions, with and without irrigation, established in the dry land areas of the Bio Bio Region. The set includes an E. nitens seed source; six E. globulus, two low, two medium and two high volumetric productivity; and three hybrids ( $E$. nitens $\times E$. globulus, $E$. camaldulensis $\times E$. globulus and E. nitens $\times$ E. camaldulensis). For this, in a sample of branches selected in the first growing season non-destructive measurements of increases and decreases absolutes and relative leaf area were made using allometric relationships established between the linear dimensions of leaf and branch, and individual and total leaf area, respectively.

The overall objective of the study was to establish the phenology and the effect of temperature and water availability on the deployment leaf in genotypes of Eucalyptus globulus, E. nitens and hybrids with E. camaldulensis.

Without irrigation was identified four periods in the deployment of photosynthetic surface, initial increase after the establishment, recess vegetative, second season growth, and leaf senescence and abscission. With irrigation, was observed the first two periods, without significant development of reactivation second season, and finally the fourth of senescence. The individuals on irrigated site were much more sensitive to climate change, absolute phase of recession which started 30 days ago. Evaluated the effect of cumulative average temperature $\left({ }^{\circ} \mathrm{C}\right)$ on the deployment of branch leaf $\left(\mathrm{cm}^{2}\right)$ groups of genotypes were identified with high and low sensitivity to low temperatures.

Keywords: Eucalyptus, Fast growth, Allometry, Water stress, Sensitivity to cold. 


\section{INTRODUCCIÓN}

La evaluación de las mejoras y respuestas genéticas de plantaciones forestales clonales es tema de discusión y perfeccionamiento constante. Los rápidos avances en la selección de la especie y genotipo a establecer centrados en superar las restricciones del sitio que limitan el crecimiento implican un aumento continuo en la eficiencia en el uso de los recursos, la productividad de los bosques, y la rentabilidad económica de la actividad forestal (Stape et al. 2004, Boyden et al. 2008, Gonçalves et al.2008).

En este sentido, la selección genética tradicional basada en la selección de individuos con características deseadas resulta poco flexible a condiciones variables del medio. Esto se debe a que la precisión de las proyecciones empíricas de productividad quedan restringidas a sitios de establecimiento particulares, siendo necesario para lograr una buena calibración de los modelos de crecimiento del seguimiento de individuos y rodales hasta edades avanzadas o final de la rotación, el empleo de variables complementarias que permitan minimizar los amplios márgenes de error de las proyecciones hechas a partir de edades tempranas (Battaglia et al.,1998; Peng 2000, Peng et al. 2002; Skovsgaard y Vanclay, 2008; Miehle et al., 2009).

Una de las variables complementarias es el área foliar (AF), ya que como indicadora de adaptabilidad a las condiciones del sitio permite aproximar parámetros tales como la productividad de biomasa de planta individual y total de rodal.

El AF puede ser determinada, modelada y relacionada en el tiempo directamente con las tasas de incremento y productividad primaria potencial volumétrica o de biomasa total (Medhurst y Beadle, 2001; Whitehead y Beadle, 2004).

El AF puede ser cuantificada en todas las etapas de crecimiento y su modelación permite cuantificar la flexibilidad y plasticidad de la especie, variedad y/o genotipo, a distintos niveles de disponibilidad de recursos y estrés. Esto debido a que el incremento foliar depende de factores determinantes como el agua disponible en el suelo, temperatura, radiación incidente, fotoperiodo y fertilidad del suelo. Tal que, en el caso de especies de rápido crecimiento, en especial en aquellas de alto interés pertenecientes a los géneros Eucalyptus y Pinus, permite comparar la eficiencia en crecimiento de genotipos e individuos establecidos en sitios contrastantes (Allen and Albaugh, 1999; Merchant el al., 2007; Boyden et al., 2008; Hubbard et al., 2010).

En el caso de plántulas de E. globulus creciendo bajo condiciones controladas se ha demostrado que una disminución en el agua disponible y un aumento de la temperatura induce la acumulación de solutos en el medio intracelular, esto modifica la turgencia restringiendo la expansión celular. Así, las consecuentes variaciones en permeabilidad y resistencia de las membranas intracelulares, plasmáticas y paredes celulares, modifican los mecanismos de difusión, y el transporte en y entre células adyacentes, lo que a nivel agregado de tejido se traduce en una diminución del crecimiento total (White et al., 2000; Wikberg and Ögren, 2007).

Como tendencia general, se ha observado que a mayor disponibilidad de recursos como agua y luz, es de esperar un mayor crecimiento y tamaño a nivel celular, de tejido y de componente de biomasa (foliar, fustal y radicular). Sin embargo, la cuantificación de la magnitud de los diferenciales de respuesta y su correlación con la productividad total de la planta en el largo plazo, en especial sobre especies y genotipos de Eucalyptus, constituye una materia de estudio incipiente (Pita and Pardos, 2001).

En clones de E. globulus se ha observado una tendencia general a que la disminución de la disponibilidad hídrica y temperatura constituyen precursores de reducción del crecimiento total de las hojas. Esta reducción sumada a un aumento de la regulación estomática inicial frente a 
condiciones de estrés hídrico constituye un mecanismo de adaptación que permite controlar la deshidratación, pero hasta un punto en que el equilibrio hídrico interno de la planta se rompe determinado cambios irreversibles de degeneración y muerte celular (Osorio et al., 1998; Guarnaschelli et al., 2006; Wikberg and Ögren, 2007).

Sin embargo, aun cuando la productividad está claramente vinculada a la intercepción lumínica y consecuentemente con la masa de follaje e índice de área foliar, a diferencia de lo ocurrido en especies de género Pinus, el conocimiento respecto al efecto de la modificación de los recursos del sitio sobre la fenología del despliegue foliar de Eucalyptus es escaso (Sampson et al., 2003; Albaugh et al., 2010).

En Eucalyptus, la gran parte de la documentación se centra en evaluaciones de carácter puntual o en intervalos de tiempo que no reflejan las variaciones interanuales del crecimiento. Del mismo modo, las diferencias inter-específicas de crecimiento están influenciadas en su mayoría por diferencias en las características edáficas de los sitos de establecimiento, lo que impide la obtención de conclusiones certeras respecto a la real tolerancia de variedades $\mathrm{y} / \mathrm{o}$ genotipos específicos a factores de estrés como la sequía, anegamiento, luminosidad, disponibilidad nutricional y salinidad (Farrel et al., 1996; Medhurst et al., 1999; White et al., 2000; Merchant et al., 2007).

En este sentido, estudios que identifiquen y cuantifiquen estrategias de crecimiento y captura lumínica similares o distintas, y su relación en el tiempo con la sensibilidad de la especie y/o genotipo de Ecualyptus a cambios disponibilidad hídrica y temperatura no han sido realizados previamente.

Por lo tanto, preguntas como cuál es el efecto del cambios en la disponibilidad hídrica del suelo sobre el patrón fenológico de despliegue foliar, cómo varía el patrón de despliegue foliar entre especies y genotipos de Eucalyptus con distintos niveles de sensibilidad frío y sequía, y cuál es la magnitud de la variación en producción foliar según especie y genotipo de Eucalyptus creciendo bajo condiciones de alta y baja disponibilidad hídrica del suelo, no han sido respondidas.

\section{OBJETIVOS}

El objetivo general del estudio fue establecer la fenología y el efecto de la disponibilidad hídrica y temperatura sobre el despliegue foliar de genotipos de Eucalyptus globulus, E. nitens e híbridos con E. camaldulensis.

Los objetivos específicos en tanto fueron:

Caracterizar la fenología foliar de Eucalyptus spp. bajo condiciones de alta y baja disponibilidad hídrica de suelo, durante las dos primeras temporadas de crecimiento.

Establecer el efecto de la disponibilidad hídrica y temperatura sobre el despliegue foliar de genotipos de Eucalyptus globulus, E. nitens e híbridos con E. camaldulensis.

\section{MATERIAL Y MÉTODO}

\section{Material Vegetal}

Se consideró un total diez genotipos de Eucalyptus (Cuadro $N^{\circ} 1$ ), correspondientes a 240 plantas viverizadas por el Programa de Mejoramiento Genético de Forestal Mininco SA, Los 
Ángeles, Chile. Las plantas fueron producidas a partir de estacas en contenedor de $110 \mathrm{~cm}^{3}$. Como sustrato se utilizó corteza de Pinus radiata D. Don compostada.

\section{Cuadro $\mathrm{N}^{\circ} 1$ \\ DESCRIPCIÓN DEL MATERIAL GENÉTICO}

\begin{tabular}{|c|c|c|c|}
\hline $\mathbf{N}^{\circ}$ & Especie & Codificación & Descripción * \\
\hline 1 & E. globulus & $\mathrm{EgA}_{1}$ & Productividad alta \\
\hline 2 & E. globulus & $\mathrm{EgA}_{2}$ & Productividad alta \\
\hline 3 & E. globulus & $\mathrm{EgM}_{1}$ & Productividad media \\
\hline 4 & E. globulus & $\mathrm{EgM}_{2}$ & Productividad media \\
\hline 5 & E. globulus & $\mathrm{EgB}_{1}$ & Productividad baja \\
\hline 6 & E. globulus & $\mathrm{EgB}_{2}$ & Productividad baja \\
\hline 7 & E. nitens & En & Semilla \\
\hline 8 & E. nitens $\times$ E. globulus & EnxEg & Clon híbrído \\
\hline 9 & E. nites $x$ E. camaldulensis & EnxEcam & Clon híbrído \\
\hline 10 & E. camaldulensis $x$ E. globulus & EcamxEg & Clon híbrído \\
\hline
\end{tabular}

\section{Descripción del Sitio de Establecimiento}

El estudio fue establecido en el Huerto Semillero Chumulco, propiedad de la empresa Forestal Mininco SA, localizado en el secano interior de la provincia del Bio Bio, Chile, entre $37^{\circ} 45^{\prime}$ LS y $72^{\circ} 18^{\prime} \mathrm{LO}, 178 \mathrm{~km}$ al sureste de la ciudad de Concepción. Sitio con clima templado cálido, estación seca y lluviosa de extensión semejantes, precipitación media anual de $1.200 \mathrm{~mm}$, temperatura media anual de $13,1^{\circ} \mathrm{C}$, media máxima mensual de $18^{\circ} \mathrm{C}$ y media mínima mensual de $7^{\circ} \mathrm{C}$. El suelo corresponde a la serie Collipulli, perteneciente al grupo de los rojo arcillosos (Alfisoles), desarrollados a partir de material piroclástico antiguo, de textura franco arcillosa a arcillosa y estructura granular a bloques subangulares, color pardo rojizo a rojizo, moderadamente profundo a profundo, muy plástico, con una gran cantidad de agua aprovechable, nivel moderado a alto de materia orgánica (4 - 8\%), acidez fuerte a moderada $(\mathrm{pH} 5-6)$ y baja densidad aparente $\left(1,01 \mathrm{~g} / \mathrm{cm}^{3}\right)$.

\section{Diseño Experimental y Mediciones}

Los diez genotipos de Eucalyptus spp. fueron establecidos en agosto de 2008, a una densidad de 1.667 plantas/ha, en dos sitios adyacentes (similares condiciones edafoclimáticas). El primero de baja disponibilidad hídrica del suelo durante el periodo estival (sin riego) y el segundo de alta disponibilidad hídrica (con riego), regulada mediante riego por goteo entre los meses de septiembre y marzo. Se consideró un diseño de bloques adyacentes completos al azar, con restricción de no adyacencia de genotipos entre bloques, en un arreglo factorial de tratamientos; 4 rametos $\times 10$ genotipos $\times 3$ bloques $\times 2$ condiciones de humedad.

Una cuantificación y comparación intensiva del despliegue foliar de los genotipos de Eucalyptus fue realizada mediante mediciones quincenales ( \pm 5 días) durante las dos primeras temporadas de crecimiento en terreno (entre el 20 diciembre de 2008 y el 05 de agosto de 2010) sobre una selección aleatoria de 60 plantas ( 1 rameto $\times 10$ genotipos $\times 3$ bloques $\times 2$ condiciones de humedad). En ellas se monitoreó en forma no destructiva el incremento y senescencia absoluta 
de la superficie foliar a nivel de hoja y agregada de rama. Se cuantificó el área foliar de la primera rama de crecimiento inmediato postestablecimiento desarrollada en orientación sobre hilera, y la primera desarrollada entre hileras.

El procedimiento no destructivo repetido en cada oportunidad de medición implicó el uso de relaciones alométricas para la estimación del área foliar a nivel de hoja individual y agregado de rama, considerando como base sus dimensiones lineales. Se cuantificó la elongación de cada hoja, en longitud máxima $(L \pm 0.1 \mathrm{~cm})$ y ancho máximo $(A, \pm 0.1 \mathrm{~cm})$. La senescencia de cada hoja fue registrada cuando el porcentaje de superficie marchita (seca) o con degradación mecánica fue mayor al $50 \%$ del área total, en su defecto se identificó la cicatriz dejada en la rama por la abscisión de la hoja.

Para la estimación del área foliar de hoja individual se utilizó el modelo básico de regresión lineal cuya variable explicativa la conformaron combinaciones de sus dimensiones (largo y ancho máximos). Los valores de AF de hoja de referencia para calibración del modelo fueron obtenidos mediante análisis fotográfico. La calibración fue ejecutada durante julio de 2009 (fase invernal de recesión del crecimiento de primera temporada) y consistió en una submuestra de follaje sin daño mecánico y/o pérdida de turgencia evidente, compuesta de 120 hojas por genotipo $(20$ hojas $\times$ rameto seleccionado $\times$ genotipo $\times$ bloque $\times$ condición), representativas del rango de distribución de tamaños de hoja observados.

El procedimiento de obtención de los valores de calibración mediante fotografías implicó la captura de una imagen digital por hoja directamente desde la planta, similar al principio óptico de la planimetría (Osorio et al., 1998). Para ello, se utilizó un cámara fotográfica Canon A1100-IS de 12,1 mega píxeles y una unidad de referencia rígida de color blanco de $30 \times 30 \mathrm{~cm}$, con cuatro referencias espaciales de $10 \mathrm{~cm}$, bajo condiciones homogéneas de perpendicularidad y luminosidad. La superficie individual de cada hoja fue aislada y determinada mediante análisis fotográfico utilizando el software SigmaScan Pro 5.0 DEMO ImageAnalysis Software (SYSTAT, Richmond, CA, USA).

Una cuantificación continúa de temperatura ambiental diaria (media, mínima y máxima, en ${ }^{\circ} \mathrm{C}$ ) fue realizada durante el periodo de estudio utilizando una estación meteorológica (WS2-550) ubicada a $250 \mathrm{~m}$ lineales del ensayo.

\section{Análisis Estadístico}

Análisis de valores foliares relativos acumulados fue utilizado para establecer gráficamente la existencia de fases en periodo de despliegue de área foliar de primera y segunda temporada de crecimiento. El efecto de la disponibilidad hídrica del suelo sobre la extensión del periodo del despliegue foliar relativo de primera temporada fue establecido por genotipo mediante modelación no lineal. Para ello, fueron establecidas diferencias significativas entre los parámetros modelo logístico [1], donde ' $a$ ' representa la tasa de incremento relativa $\left(I_{\max }\right)$, y ' $b$ ' representa la escala de tiempo relativo o periodo de tiempo relativo requerido para lograr el $100 \%$ del despliegue foliar $\left(T_{\max }\right)$.

$$
y=\frac{100}{\left(1+b e^{-a x}\right)}
$$

Donde: $y$ : variable explicada (incremento relativo de área foliar media de rama, \%)

$x$ : variable explicatoria (tiempo relativo, \%) 
a y $b$ : parámetros de escala y forma $(x \geq a, b>0)$, y e: base

de los logaritmos naturales.

Similar análisis fue realizado para determinar el efecto de la temperatura media acumulada $\left({ }^{\circ} \mathrm{C}\right)$ sobre el despliegue foliar de rama $\left(\mathrm{cm}^{2}\right)$. Para ello, fueron incluidos como variable explicatoria en el modelo [1] los valores de temperatura media acumulada relativa del total de temperatura acumulada durante la primera temporada de crecimiento.

La totalidad de los análisis fue realizada utilizando el software estadístico SAS 9.1 (SAS Institute Inc., NC, USA, 2002).

\section{RESULTADOS}

Evaluado el efecto de la disponibilidad hídrica del suelo sobre el incremento foliar acumulado fueron identificadas fases en el despliegue foliar por condición. Con riego, se identificaron tres fases i) incremento inicial, ii) receso vegetativo invernal, y iii) senescencia y abscisión.

Como tendencia general, para todos los genotipos, la fase i) se extendió desde diciembre de 2008 (primera medición) hasta mayo de 2009, los genotipos En y EnxEc, culminaron el incremento inicial anticipadamente (abril 2009). Culminada la fase ii) se observó la pérdida del follaje acumulado sobre todos los genotipos analizados (Figura $\mathrm{N}^{\circ} 1$ ).

Sin riego, se identifió cuatro fases en el despliegue foliar i) incremento inicial, ii) recesión vegetativa invernal, iii) incremento de segunda temporada y iv) senescencia y abscisión. Sobre los genotipos En y EnxEc se observó una mayor extensión de la fase de incremento inicial, cuya diferencia resultó superior a los 30 días (20 mayo 2009 - 05 junio 2009), respecto a la condición con riego. Todos los genotipos evaluados presentaron incremento de segunda temporada (Figura $\left.N^{\circ} 1\right)$.

En ambas condiciones de crecimiento el genotipo EcxEg resultó altamente sensible a las condiciones térmicas del sitio. Con riego, perdió el $90 \%$ follaje acumulado de primera temporada, entre junio y octubre de 2009. En similar periodo, sin riego, perdió el $100 \%$ del follaje acumulado. La pérdida del 10\% restante, con riego, ocurrió entre marzo y mayo de 2010 (Figura № 1).

Evaluado el incremento relativo de área foliar de primera temporada de crecimiento por genotipo y condición, relativizado respecto al periodo de tiempo comprendido entre la primera medición (20 diciembre 2008) y la última medición de primera temporada de crecimiento (05 junio 2009), se identificó grupos de genotipos con similar tasa de incremento relativo $\left(I_{\max }\right)$ (parámetro a en el modelo [1]), y similar escala de tiempo relativo ( $T_{\max }$ ) (parámetro $b$ en el modelo [1]) (Cuadro $\mathrm{N}^{\circ}$ 2).

Con riego, los genotipos EgA1, EgM1, EgM2, EgB2, En, EnxEg, EnxEc conformaron el Grupo "a" con similar $I_{\max }$, seguidos de los genotipos EgA2 y EgB1 (Grupo "b"), el genotipo EcxEg (Grupo "c") presentó la mayor tasa de incremento relativa foliar. Sobre el $T_{\max }$ se identificaron dos grupos, el primero conformado por los genotipos EgA1, EgA2, EgM2, En, EnxEg y EncEc, y el segundo conformado por los genotipos EgM1, EgB1, EgB2 y EcxEg (Cuadro № 2).

Sin riego, se observó un gran grupo de genotipos con similar $I_{\max }$, solo EnxEc resultó significativamente inferior al resto (Cuadro $\mathrm{N}^{\circ} 2$ ). 
Evaluado el $T_{\max }$ se estableció la existencia de tres grupos de genotipos, el primero conformado por el genotipo EgM2 y EnxEc, el segundo por EgA1, EgA2, EgM1, EgB1, EnxEg y EcxEg, y el tercero compuesto por EgB2 y En. Entre condiciones de disponibilidad hídrica los genotipos EgA2, EgM1, EgB1, EgB2, EnxEg, EnxEc y EcxEg variaron en $I_{\max }$, mientras que EgM1, EgM2, EgB1, EgB2 y EcxEg variaron en $T_{\max }$ (Cuadro $\mathrm{N}^{\circ} 2$ ).

Evaluado el efecto de la temperatura media acumulada sobre el incremento relativo de área foliar media de rama se repitieron las tendencias observadas respecto al tiempo. Con riego, los genotipos En y EnxEc denotaron una menor temperatura media acumulada para lograr el máximo incremento foliar versus la condición sin riego.

La diferencia fue cercana a los $450^{\circ} \mathrm{C}$, equivalentes al $15 \%$ (aprox.) del total de temperatura media acumulada durante la fase de crecimiento inmediato (Figura $\mathrm{N}^{\circ} 1$ ).

Culminado el crecimiento de primera temporada del genotipo EgA1, a similar temperatura acumulada, el incremento relativo de área foliar con riego fue cerca de un $20 \%$ mayor a lo alcanzado en condición sin riego. Sin embargo, en términos absolutos la diferencia foliar fue aproximadamente de 2.264,4 $\mathrm{cm}^{2} / \mathrm{rama}$, siendo que con riego el máximo alcanzado fue de 2.994,2 $\mathrm{cm}^{2} /$ rama. En el caso de los genotipos EgA2, EgM1, EgM2, EgB1, EgB2, En, EnxEg, EnxEc y EcxEg, las diferencias absolutas fueron de 731,1; 3.306,2; 2.190,5; 3,948,0; 4.455,1; $4.222,7 ; 2.744,3 ; 1.477,8$ y $3.508,6 \mathrm{~cm}^{2} / \mathrm{rama}$, respectivamente, de un total alcanzado con riego de $1.595,7 ; 3.793,0 ; 2.785,8 ; 4.64,6 ; 5.041,6 ; 5.216,4 ; 4.073,8 ; 2.412,4$ y $4.319,4 \mathrm{~cm}^{2} / \mathrm{rama}$, respectivamente.

\section{Cuadro $\mathrm{N}^{\circ} 2$ \\ PARÁMETROS DEL MODELO DE CRECIMIENTO LOGÍSTICO [1] AJUSTADO PARA EL DESPLIEGUE DE ÁREA FOLIAR RELATIVA MEDIA DE RAMA DURANTE LA PRIMERA TEMPORADA CRECIMIENTO.}

\begin{tabular}{|c|c|c|c|c|}
\hline \multirow{3}{*}{ Genotipo } & \multicolumn{4}{|c|}{ Condición } \\
\hline & \multicolumn{2}{|c|}{ Con riego } & \multicolumn{2}{|c|}{ Sin riego } \\
\hline & $a$ & $b$ & $a$ & $b$ \\
\hline EgA1 & $0,06578^{*} \mathrm{cA}$ & $7,72240^{*} \mathrm{bA}$ & $0,06160^{*} a A$ & $8,76680^{*} \mathrm{bA}$ \\
\hline $\mathrm{EgA} 2$ & $0,08198^{*} \mathrm{bA}$ & $12,1485^{*} \mathrm{bA}$ & $0,06206^{*} \mathrm{aB}$ & $9,08190^{*} \mathrm{bA}$ \\
\hline EgM1 & $0,07292^{*} \mathrm{cA}$ & $19,3840^{*}$ aA & $0,05626^{*} \mathrm{aB}$ & $9,75060^{*} \mathrm{bB}$ \\
\hline EgM2 & $0,06685^{*} \mathrm{cA}$ & $10,8038^{*} \mathrm{bA}$ & $0,05958^{*} a A$ & $4,78410^{*} \mathrm{cB}$ \\
\hline EgB1 & $0,08532^{*} \mathrm{bA}$ & $35,8181^{*} a A$ & $0,05821^{*} a B$ & $10,0996^{*} b B$ \\
\hline EgB2 & $0,08119^{*} \mathrm{cA}$ & $28,7926^{*}$ aA & $0,06127^{*} \mathrm{aB}$ & $15,7874^{*} \mathrm{aB}$ \\
\hline En & $0,07740^{*} \mathrm{cA}$ & $12,5275^{*} \mathrm{bA}$ & $0,07005^{\star} \mathrm{aA}$ & $18,7487^{*} a A$ \\
\hline EnxEg & $0,07948^{*} \mathrm{cA}$ & $13,4737^{*} \mathrm{bA}$ & $0,05707^{\star} \mathrm{aB}$ & $10,7583^{*} \mathrm{bA}$ \\
\hline EnxEc & $0,06675^{\star} \mathrm{cA}$ & $9,67140^{*} \mathrm{bA}$ & $0,04890^{*} \mathrm{bB}$ & $5,31230^{*} \mathrm{cA}$ \\
\hline EcxEg & $0,10985^{*} \mathrm{aA}$ & $51,6801^{*} \mathrm{aA}$ & $0,06731^{*} \mathrm{aB}$ & $9,33660^{*} \mathrm{bB}$ \\
\hline
\end{tabular}

Modelo [1]: $y=100 /\left(1+b^{*} \exp \left(-a^{*} x\right)\right)$

Donde: $\quad y$ : área foliar relativa acumulada durante la primera temporada de crecimiento (\%). $x$ : día de medición relativo respecto al periodo comprendido entre la primera medición (20-12-2008) y la ultima medición de primera temporada de crecimiento (05-06-2009) $a$ y $b$ : parámetros de ajuste (tasa de incremento, y escala de tiempo, respectivamente).

exp: inversa del logaritmo natural.

Letras minúsculas distintas indican deferencias de parámetro entre genotipos por condición de disponibilidad hídrica.

Letras mayúsculas distintas indican deferencias de parámetro por genotipo entre condiciones de disponibilidad hídrica.

* Significativo (95\% de confianza). 

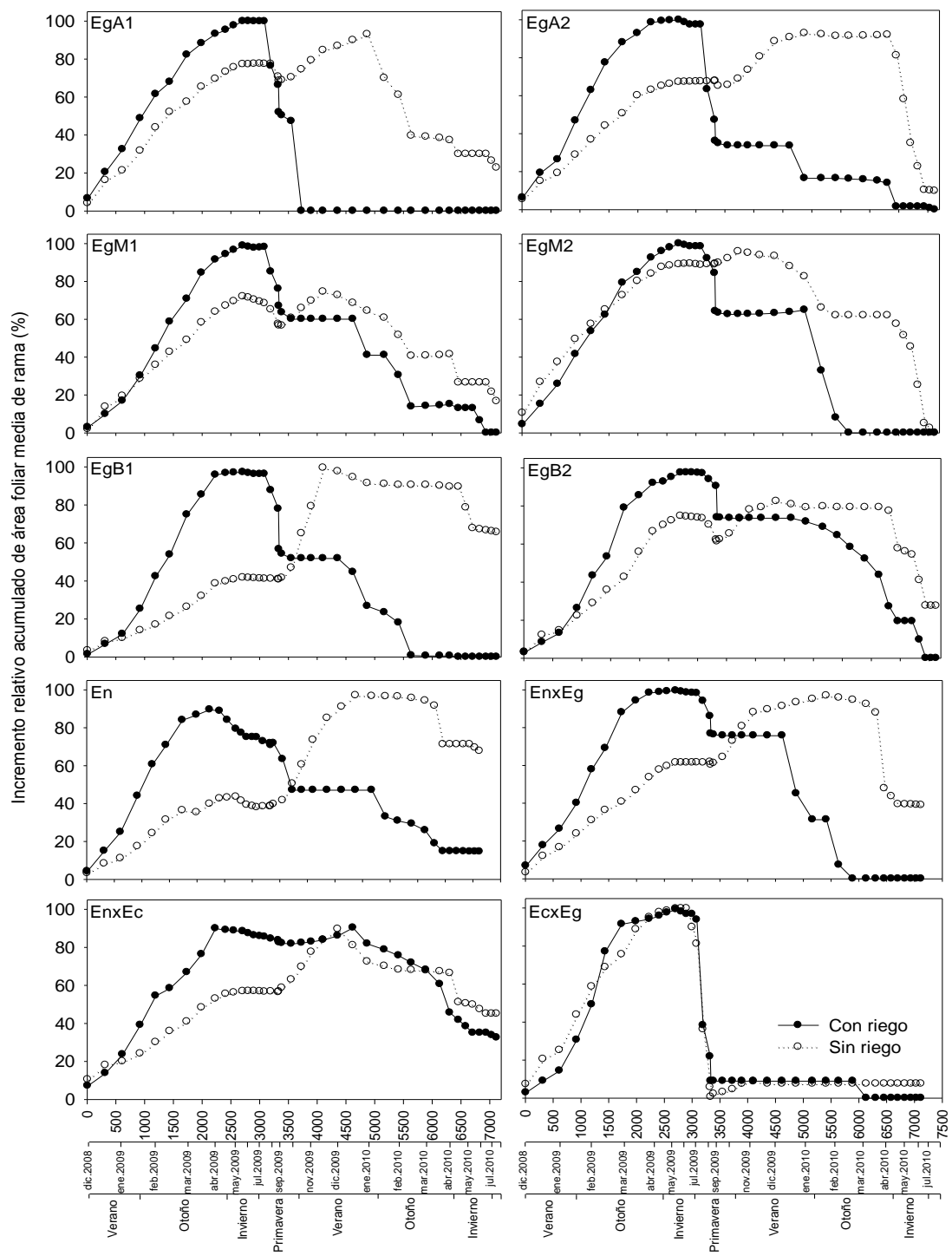

Temperatura media diaria acumulada $\left({ }^{\circ} \mathrm{C}\right)$

Fecha de medición (mm-aa)

$$
\text { Estacion del año }
$$

Figura $\mathbf{N}^{\circ} 1$

INCREMENTO RELATIVO DE ÁREA FOLIAR DE Eucalyptus spp. DURANTE LA PRIMERA Y SEGUNDA TEMPORADA DE CRECIMIENTO, BAJO CONDICIONES DE ALTA Y BAJA DISPONIBILIDAD HÍDRICA DEL SUELO (CON Y SIN RIEGO). 
Evaluado el incremento relativo de área foliar medio de rama respecto a la temperatura acumulada media diaria relativizada al total de temperatura acumulada durante la fase de incremento inicial, con riego, para ambos parámetros del modelo [1] fueron identificados los mismos grupos de genotipos establecidos previamente respecto al periodo de tiempo relativo $\left(T_{\max }\right)$. Solo los genotipos EgA2 y EgB2 se intercambiaron de grupo de $I_{\max }$ (parámetro a).

Del mismo modo, entre condiciones de disponibilidad resultaron similares los genotipos que variaron en tasa de incremento relativa, solo los genotipos EgM2, EgB1 y EcxEg, variaron significativamente en temperatura máxima relativa necesaria para alcanzar el $100 \%$ incremento foliar de primera temporada (Cuadro $\mathrm{N}^{\circ} 3$ ).

\section{Cuadro $\mathrm{N}^{\circ} 3$ \\ PARÁMETROS DEL MODELO DE CRECIMIENTO LOGÍSTICO [1] AJUSTADO PARA EL DESPLIEGUE DE ÁREA FOLIAR RELATIVA MEDIA DE RAMA DE PRIMERA TEMPORADA DE CRECIMIENTO SEGÚN TEMPERATURA MEDIA ACUMULADA}

\begin{tabular}{|c|c|c|c|c|}
\hline \multirow{3}{*}{ Genotipo } & \multicolumn{4}{|c|}{ Condición } \\
\hline & \multicolumn{2}{|c|}{ Con riego } & \multicolumn{2}{|c|}{ Sin riego } \\
\hline & $a$ & $b$ & $a$ & $b$ \\
\hline EgA1 & $0,057479^{\star} \mathrm{bA}$ & $8,20730^{*} \mathrm{bA}$ & $0,054820^{*} a A$ & $9,63550^{*} \mathrm{bA}$ \\
\hline $\mathrm{EgA} 2$ & $0,070912^{*} \mathrm{bA}$ & $12,8741^{*} \mathrm{bA}$ & $0,055299^{*} \mathrm{aB}$ & $10,0232^{*} \mathrm{bA}$ \\
\hline EgM1 & $0,065195^{\star} \mathrm{bA}$ & $22,3516^{*} a A$ & $0,051248^{*} a B$ & $11,1679^{*} \mathrm{bA}$ \\
\hline EgM2 & $0,059167^{\star} \mathrm{bA}$ & $11,8755^{*} \mathrm{bA}$ & $0,052074^{*} \mathrm{aA}$ & $5,01520^{*} \mathrm{cB}$ \\
\hline EgB1 & $0,075818^{*} \mathrm{aA}$ & $41,8791^{*} \mathrm{aA}$ & $0,052910^{*} a B$ & $11,5843^{*} b B$ \\
\hline EgB2 & $0,071938^{*} \mathrm{aA}$ & $33,1406^{*}$ aA & $0,056636^{*} \mathrm{aB}$ & $19,3007^{*} \mathrm{aA}$ \\
\hline En & $0,067164^{*} \mathrm{bA}$ & $13,3302^{*} b A$ & $0,063433^{*} \mathrm{aA}$ & $22,2803^{*} a A$ \\
\hline EnxEg & $0,069242^{*} \mathrm{bA}$ & $14,5235^{*} \mathrm{bA}$ & $0,052454^{*} \mathrm{aB}$ & $12,6186^{*} \mathrm{bA}$ \\
\hline EnxEc & $0,059058^{*} \mathrm{bA}$ & $10,5643^{*} \mathrm{bA}$ & $0,044226^{*}$ bB & $5,81370^{*} \mathrm{cA}$ \\
\hline EcxEg & $0,094623^{*} a A$ & $55,6283^{*} a A$ & $0,059265^{*} \mathrm{aB}$ & $10,1149^{*} \mathrm{bB}$ \\
\hline
\end{tabular}

Modelo [1]: $y=100 /\left(1+b^{*} \exp \left(-a^{*} x\right)\right)$

Donde: $\quad y$ : área foliar media de rama relativa acumulada durante la primera temporada (\%).

$x$ : día de medición relativo respecto al periodo comprendido entre la primera medición (20-12-2008) y la ultima medición de primera temporada de crecimiento (05-06-2009).

a y $b$ : parámetros de ajuste (tasa de incremento, y escala de temperatura, respectivamente).

exp: inversa del logaritmo natural.

Letras minúsculas distintas indican deferencias de parámetro entre genotipos por condición de disponibilidad hídrica.

Letras mayúsculas distintas indican deferencias de parámetro por genotipo entre condiciones de disponibilidad hídrica.

* Significativo (95\% de confianza).

\section{DISCUSIÓN}

El efecto significativo de la disponibilidad hídrica y temperatura sobre la fenología del despliegue relativo y absoluto de área foliar observado es coincidente con lo descrito para el género en su lugar de origen. Tal que, en plantaciones de $E$. globulus y $E$. nitens establecidas en Australia han sido observadas diferencias significativas en el índice de área foliar atribuibles a la interacción de los factores disponibilidad hídrica y temperatura media anual (Bataglia et al., 1996, Bataglia et al., 1998). 
Sin embargo, las fases fenológicas del despliegue foliar entre la sabana Australiana (Región de Darwin) y el sitio de estudio (secano interior, Región del Bio Bio, Chile) son disímiles. En el primer caso, de describe la existencia de un patrón de despliegue foliar compuesto por una fase de pleno crecimiento durante el periodo de transición entre la estación seca y la lluviosa, seguida de una fase de recesión de crecimiento durante la época de mayor precipitación, y una fase que concentra la pérdida de follaje a mediados de la estación seca siguiente, fenología de crecimiento típica de plantas de climas tropicales. Mientras que en el segundo caso (sin riego), se describen cuatro fases regulares (i., ii., iii., y iv.) cuyos limites de extensión se explican por las variaciones climáticas regulares típicas de climas templado-cálidos.

En el sitio de estudio una disminución de las temperaturas medias acumuladas (acumulación de temperaturas medias más bajas) asocian una disminución de los incrementos foliares, e inicio de los periodos de recesión vegetativos de primera y segunda temporada de crecimiento (Figura $\mathrm{N}^{\circ}$ 1). La inexistencia de una segunda temporada de incremento foliar bajo condiciones del alta disponibilidad hídrica (con riego) se explica por el crecimiento exponencial de la planta completa, observándose luego de la fase de recesión vegetativa invernal una concentración de la actividad meristemática en los estratos superiores del dosel, los que concentran la captura lumínica disminuyendo e inhibiendo la actividad fotosintética del follaje inferior, el cual inicia su abscisión.

Las diferencias generales observadas respecto extensión del periodo de tiempo requerido por los genotipos En y EnxEc para desplegar el $100 \%$ del AF resultan coincidentes con las observadas en otras especies de Eucalyptus. Tal que, en individuos de $E$. miniata y $E$. tetrodonta han sido cuantificadas diferencias significativas en la longitud del periodo de tiempo requerido para desplegar el máximo de área foliar, comenzando anticipadamente y siendo más corto en el primer caso (Williams et al. 1997). Del mismo modo, las diferencias absolutas en incremento foliar acumulado total observadas entre En y los restantes genotipos coincide con un mayor crecimiento previamente descrito para la especie bajo similares condiciones de temperaturas comparado con E. globulus, el cual ha sido caracterizado con una mayor incidencia de daño por helada (Bataglia et al., 1996, Bataglia et al., 1998).

Del mismo modo, la reducción y homogeneidad de las tasas de incremento relativas observadas durante la fase de crecimiento inmediato sin riego denotan una adaptación significativa y general de los genotipos evaluados a las condiciones de disponibilidad hídrica (Cuadro $\mathrm{N}^{\circ} 2$ ). Esto favorecen la minimización de la resistencia de la copa al atenuar el efecto desecante del viento mediante la reducción de la superficie foliar agregada de rama, que combinada con un aumento cuticular, aumenta la eficiencia de la disipación de la temperatura cuando la radiación incidente es máxima, favoreciendo la mantención del estado hídrico (Farrell et al. 1996, King 1999, Allen, 2000, Chaves et al., 2003, Allen et al., 2005).

Por otra parte, la existencia de tres grupos disimiles en $T_{\max }$, observados sin riego, es reflejo directo de variaciones en susceptibilidad de los genotipos integrantes de cada grupo a las variaciones térmicas del sitio, lo cual se valida establecer una igual conformación de grupos cuando el incremento relativo de área foliar se explica en función de la temperatura media acumulada (Cuadros $\mathrm{N}^{\circ} 2$ y N³).

\section{CONCLUSIONES}

Un aumento en la disponibilidad hídrica del suelo favorece un aumento en el incremento de área foliar absoluta $\left(\mathrm{cm}^{2}\right)$ media de rama de Eucalyptus. Bajo condiciones de alta disponibilidad de agua en el suelo se observa solo una temporada de incremento foliar en ramas de primera 
temporada, mientras que bajo condiciones de sequia se observa una reactivación del incremento de segunda temporada.

Bajo las condiciones de sitio analizadas es posible observar un efecto positivo de la disponibilidad hídrica sobre la tasa de incremento relativo de área foliar media de rama (\%). Mientras que una reducción el periodo de tiempo y temperatura media diaria acumulada necesaria para alcanzar el máximo foliar se observa como respuesta particular según genotipo analizado, pudiendo ser identificados aquellos que requieren menor tiempo y menor temperatura acumulada para desplegar el $100 \%$ de la superficie por temporada de crecimiento.

\section{RECONOCIMIENTOS}

El financiamiento para este estudio fue otorgado por el Fondo Nacional de Desarrollo Científico y Tecnológico, Proyecto FONDECYT 1085093. Se agradece la colaboración otorgada por Forestal Mininco SA. Se agradece el financiamiento de CONICYT por la beca otorgada para estudios de Magister en Chile (MP). Se agradece el apoyo brindado por la Facultad de Ciencias Forestales de la Universidad de Concepción y de la Cooperativa de Productividad Forestal (FPC).

\section{REFERENCIAS}

Albaugh, T., Allen, L ., Stape, J., Fox, T., Rubilar, R., Carlson, C. and Pezzutti, R., 2010. Leaf area duration in natural range and exotic Pinus taeda. Canadian Journal of Forest Research 40: 224-234.

Allen, H, Albaugh, T., 1999. Ecophysiological basis for plantation production: A Loblolly Pine case study. Bosque 20(1): 3-8.

Allen, R. G., 2000. Using the FAO-56 dual crop coefficient method over an irrigated region as part of an evapotraspiration intercomparason study. Journal of Hydrology 229: 27-41.

Allen, R., Pereira, L., Smith, M., Raes, D. and Wright, J., 2005. FAO-56 Dual Crop Coefficient Method for Estimating Evaporation from Soil and Application Extensions. Jurnal of Irrigation and Drainage Engineering131: 213.

Battaglia, M., Beadle, C. and Loughhead, C., 1996. Photosynthetic temperature responses of Eucalyptus globulus and Eucalyptus nitens. Tree Physiology 16: 81-89.

Battaglia, M., Cherry, M., Beadle, L., Sands, J. and Hingston, A., 1998. Prediction of leaf area index in Eucalypt plantations: Effects of water stress and temperature. Tree Physiology 18: 521-528.

Boyden, S, Binkley, D. and Stape, J., 2008. Competition among Eucalyptus trees depends on genetic variation and resource supply. Ecology 89(10): 2850-2859.

Chaves, M., Maroco, J. and Pereira, J., 2003. Uderstanding plant responses to drought- from genes to the whole plant. Functional Plant Biology 30: 239-264

Farrell, R., Bell, D., Akilan, K. and Marshall, J., 1996. Morphological and Physiological Comparisons of Clonal Lines of Eucalyptus camaldulensis. I. Responses to Drought and Waterlogging. Australian Journal Plant Physiology 23: 497-507.

Gonçalves, J., Stape, J., Laclau, J., Bouillet, J. and Ranger, J., 2008. Assessing the effects of early silvicultural management on long-term site productivity of fast-growing Eucalypt plantations: the Brazilian experience. Southern Forests 70(2): 105-118. 
Guarnaschelli, A, Prystupa, P. and Lemcoff, J., 2006. Drought conditioning improves water status, stomatal conductance and survival of Eucalyptus globulus subsp. bicostata seedlings. Annals of forest Science 63: 941950.

Hubbard. R, Stape, J., Ryan, M., Almeida, A . and J Rojas, J., 2010. Effects of irrigation on water use and water use efficiency in two fast growing Eucalyptus plantations. Forest Ecology and Management 259: 17141721.

King, D., 1999. Juvenile Foliage and the Scaling of Tree Proportions, with Emphasis on Eucalyptus. Ecology 80(6): 1944-1954.

Medhurst, J., Battaglia, M., Cherry, M., Hunt, M., White, D. and Beadle, C., 1999. Allometric relationships for Eucalyptus nitens (Deane and Maiden) Maiden plantations. Trees 14: 91-101.

Medhurst, J. and, Beadle, C., 2001. Crown structure and leaf area index development in thinned and unthinned Eucalyptus nitens plantations. Tree Physiology 21: 989-999.

Merchant, A., Callister, A., Arndt, S., Tausz, M. and Adams, M., 2007. Contrasting Physiological Responses of Six Eucalyptus Species to Water Deficit. Annals of Botany 100: 1507-1515.

Miehle, P., Battaglia, M., Sands, P., Forrester, D., Feikema, P., Livesley, S ., Morris, J. and Arndt, S., 2009. A comparison of four process-based models and a statistical regression model to predict growth of Eucalyptus globulus plantations. Ecological Modelling 220: 734-746.

Osório J, Osório, M., Chaves, M. and Pereira, J., 1998. Water deficits are more important in delaying growth than in changing patterns of carbon allocation in Eucalyptus globulus. Tree Physiology 18: 363-373.

Peng, C., 2000. Growth and yield models for uneven-aged stands: past, present and future. Forest Ecology and Management 132: 259-279.

Peng, C, Liu, J., Dang, Q., Apps, M. and Jiang, H., 2002. TRIPLEX: A generic hybrid model for predicting forest growth and carbon and nitrogen dynamics. Ecological Modelling 153: 109-130.

Pita, P., and Pardos, J., 2001. Growth, leaf morphology, water use and tissue water relations of Eucalyptus globulus clones in response to water deficit. Tree Physiology21: 599-607.

Sampson, D., Albaugh, T., Johnsen, K., Allen, H. and Zarnoch, S., 2003. Monthly leaf area index estimates from point in time measurements and needle phenology for Pinus taeda. Canadian Journal of Forest Research 33 : 2477-2490.

Skovsgaard, J. and Vanclay, J., 2008. Forest site productivity: A review of the evolution of dendrometric concepts for even-aged stands. Forestry 81(1): 13-29.

Stape, J., Binkley, D. and Ryan, M., 2004. Eucalyptus production and the supply, use and the efficiency of use of water, light and nitrogen across a geographic gradient in Brazil. Forest Ecology and Management 193: 17-31.

White, D., Turner, N. and Galbraith, J., 2000. Leaf water relations and stomatal behavior of four allopatric Eucalyptus species planted in Mediterranean southwestern Australia. Tree Physiology 20: 1157-1165.

Whitehead, D. and Beadle, C., 2004. Physiological regulation of productivity and water use in Eucalyptus: A review. Forest Ecology and Management 193: 113-140.

Wikberg, J. and Ögren, E., 2007. Variation in drought resistance, drought acclimation and water conservation in four willow cultivars used for biomass production. Tree Physiology 27: 1339-1346

Williams, J., Myers, B., Muller, W., Duff, G. and Eamus, D., 1997. Leaf Phenology of Woody Species in a North Australian Tropical Savanna. Ecology 78(8): 2542-2558. 
